\title{
Amplitude variations in the sdBV star PG 1605+072: Another beating time scale?^
}

\author{
T. M. D. Pereira ${ }^{1}$ and I. P. Lopes ${ }^{1,2}$ \\ ${ }^{1}$ Centro Multidisciplinar de Astrofísica, Instituto Superior Técnico, Av. Rovisco Pais, 1049-001 Lisboa, Portugal \\ e-mail: tiago.pereira@ist.utl.pt \\ 2 Department of Physics, Denys Wilkinson Building, Keble Road, Oxford University, OX1 3RH, UK \\ e-mail: lopes@astro.ox.ac.uk
}

Received 3 March 2004 / Accepted 3 May 2004

\begin{abstract}
PG 1605+072 has an unique and complex oscillation spectrum amongst the pulsating members of the EC 14026 stars. It has the longest periods and the richest, most puzzling frequency spectrum. We present a quantitative analysis of the photometric time-series obtained at 1-m telescope of the South African Astronomical Observatory. Thirteen oscillation parameters, frequencies, amplitudes and initial phases were determined from a $45 \mathrm{~h}$ time-series. Our work confirm previous observational results. The observed frequencies are within a difference smaller than $2.7 \%$ of the theoretical values, and less than $0.1 \%$ of other previous studies. We also infer the existence of variation of a periodicity of 4-5 days on the amplitude of the observed modes, similar to the yearly time-scale variation found by previous studies. Furthermore, we found a new frequency of $2133 \mu \mathrm{Hz}$ which has not been previously reported, its origin being yet unclear.
\end{abstract}

Key words. stars: subdwarfs - stars: oscillations - stars: individual: PG 1605+072 - stars: horizontal branch

\section{Introduction}

The hot sub luminous dwarf B stars, usually refereed as sdB stars, form a homogeneous group dominating the stellar population of faint blue stars in our own Galaxy. These stars are believed to be the most important source of UV radiation excess, observed in older galaxies (Brown et al. 1997, 2000), usually known as UV upturn. The formation and evolution of sdB stars in different stellar evolution channels, as an isolated object or within a binary system is still open to debate, being of fundamental importance to our understanding of the evolution of galaxies. Moreover, the use of sdBs as standard candles for the age determination of old galaxies makes this study even more urgent.

Subdwarf B (sdB) stars are evolved, low mass, core-helium burning stars with a thin hydrogen envelope. These hot stars are believed to be extreme horizontal branch (EHB) objects (Saffer et al. 1994), and will later evolve to the O type (sdO), ultimately joining the white dwarf cooling track. Although they descend from the red giant branch, the physical processes that originate their evolution towards the EHB are not clear.

Nevertheless, the perspectives for the study of sdBs have been greatly improved with the discovery of pulsating sdB stars

* Based on observations obtained at the South African Astronomical Observatory (SAAO). This research was supported by a grant from Fundação da Ciência e Tecnologia, grant No. PESO/P/PRO/40142/2000.
(Kilkenny et al. 1997). The possibility to probe the interior of sdB stars with asteroseismological tools has motivated several studies throughout the years. Nowadays there are more than 40 known pulsating sdBs, and the number keeps increasing.

The first step to use asteroseismology in sdB stars is to identify the pulsation modes (e.g. Charpinet et al. 2003). This consists in measuring the mode frequencies and with a theoretical pulsation model identify the associated quantic numbers, radial order $n$, degree $l$ and azimutal number $m$, as predicted by the linear theory of stellar pulsations. Meanwhile, we still have some way to go in the understanding the pulsating mechanism on these stars (e.g. Fontaine et al. 2003).

From the known pulsating sdBs, PG $1605+072$ has the most extreme properties: the longest periods (some above $500 \mathrm{~s})$; the lowest surface gravity $(\log g=5.25 \pm 0.10)$ and the highest number of pulsation modes. These properties, especially the long periods, make PG $1605+072$ an ideal target for observational astroseismological studies.

In the past, PG $1605+072$ has been observed many times (Koen et al. 1998; Kilkenny et al. 1999; O'Toole et al. 2000, 2002; Woolf et al. 2002; Falter et al. 2003), using either photometry (pulsations produce luminosity variations) or spectroscopy (shifts in line indices). Our work is another photometric monitoring of the variation of frequencies and amplitudes for PG $1605+072$. These observations were part of the largest spectroscopic and photometric campaign (to date) on PG $1605+072$ - the Multi Site 
Table 1. Photometic observations of PG $1605+072$.

\begin{tabular}{lll}
\hline \hline UT date & $\begin{array}{l}\text { Starting time } \\
\text { (JD 2 452 400+) }\end{array}$ & $\begin{array}{l}\text { Run length } \\
\text { (Hours) }\end{array}$ \\
\hline 2002 May 15-16 & 10.272 & 6.13 \\
2002 May 16-17 & 11.200 & 7.01 \\
2002 May 17-18 & 12.197 & 6.99 \\
2002 May 18-19 & 13.199 & 6.99 \\
2002 May 19-20 & 14.198 & 6.09 \\
2002 May 20-21 & 15.197 & 5.87 \\
2002 May 26-27 & 21.193 & 6.14 \\
\hline Total & & 45.22 \\
\hline
\end{tabular}

Spectroscopic Telescope (MSST), which coordinated several telescopes around the world, aimed to produce a long, highquality continuous time series for that star. While waiting for the full time-series analysis of the MSST campaign, our aim in this paper is to report on the new results obtained from frequency determination and the amplitude variation of the observed photometric spectrum of PG $1605+072$ obtained at the SAAO site.

\section{Observations and reductions}

The observations were performed using the UCT CCD attached to the 1-m telescope at the Sutherland site of SAAO. As a part of the MSST observational campaign of PG 1605+072 (Heber et al. 2003), we were awarded two weeks of observing time from May 13th to 26th, 2002. Bad weather conditions prevented the observations during the first two nights and during five nights in the second week. Overall, we observed during half of the allocated time, as expected in such observation site.

The observation log presented in Table 1 was obtained in several photometric runs with a integration time for each frame of $20 \mathrm{~s}$, obtained sporadically over an interval of 7 days. The total run time is $45 \mathrm{~h}$. The frame transfer mode in the UTC CCD allowed continuous acquisition with no dead times - while one half of the CCD was being readout, the other was being exposed. A BG39 filter was used, because our object has a spectral peak in the blue region, and to better combine (in the future) our data with photomultiplyer data (see O'Donoghue et al. 2000).

The reduction of the observational data was carried out by using standard techniques. For each run the frames were corrected using normalized flat fields.

The magnitudes of the target star were corrected by using differential photometry with the comparison star. A nearby comparison star, known to be a non-variable star was kept in the same frame as PG $1605+072$. Using the magnitude of the fixed star we were able to correct the magnitude of the target star from the atmospheric and the airmass effects, among other effects. This procedure was done by fitting a PSF to the objects in each frame, by using the DuPHOT/DoPHOT (Schechter et al. 1993) reduction package kindly provided to us by Darragh O’Donoghue.

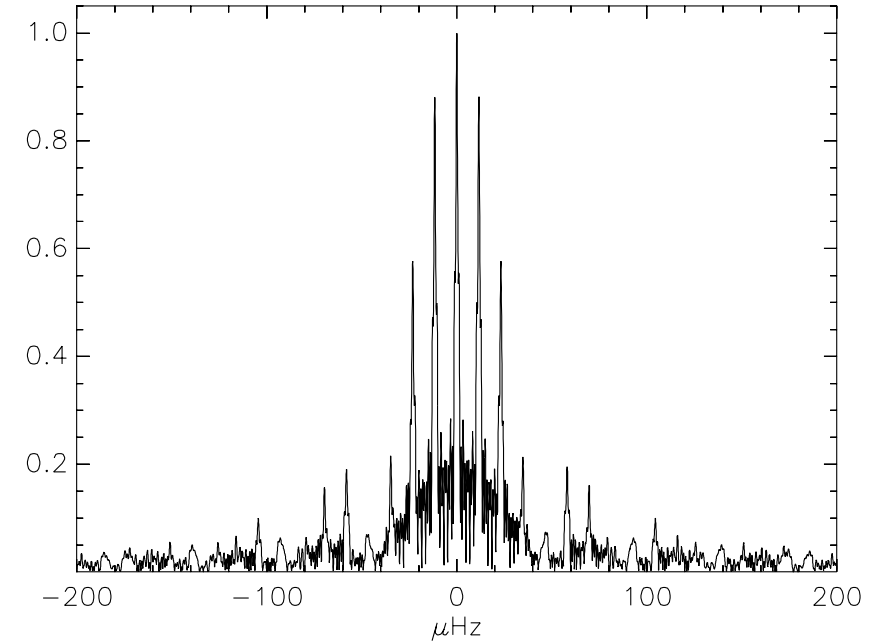

Fig. 1. Window function for the 7-night combined dataset. The function is normalized to unit and zero frequency.

\section{Time-series analysis}

The frequency analysis described in this section were performed using a least-squares sine fitting procedure. Once the light curves were reduced, our next step was to extract the frequencies, amplitudes and phases from the light curves. For each night, the formal frequency resolution was $\delta v \approx 1 / T \approx$ $43 \mu \mathrm{Hz}$ - which is quite low if we expect to accurately resolve most of the peaks in the $1800-2500 \mu \mathrm{Hz}$ interval. The frequency resolution was improved by combining the data from the seven nights, at the expense of introducing aliasing into the amplitude spectrum. The combined data yield a measured resolution of approximately $3.15 \mu \mathrm{Hz}$, which is the best these observations allow. Unfortunately, the combination of the night runs of data with day time observing gaps introduces the aliasing peaks. In the observed spectrum several alias peaks appear around each true peak. This is the so-called side-band structure, that can be easily seen in our window function (cf. Fig. 1).

After trials with different methods, we found that the leastsquares sine fitting, was the best strategy to estimate the frequencies from the 7-night combined light curve. The leastsquares sine fitting program Period98 (Sperl 1998) was used to calculate the parameters. One sinusoid was fitted at each time, and the procedure repeated several times - without subtracting the previously adjusted sinusoid (pre-whitening).

Although pre-whitening allows a "cleaner" light curve, one has a problem if the fitted sinusoid does not correspond to a very good fit. Because of the errors (especially in the phase parameter space) due to the fitting process, subtracting a sinusoid with a incorrect initial phase could result in erroneous data added to the light curve. This problem becomes more important as we have several closely spaced frequencies in the spectrum, and incorrect subtractions could yield poor results. To avoid this problem, and after different trials, it was decided not to subtract the fitted frequencies, and in that way we obtain a better estimation of the oscillation parameters.

A total of 13 frequencies were extracted. We could have probably detected more frequencies using a different method, but our emphasis was to determine the frequencies with the 


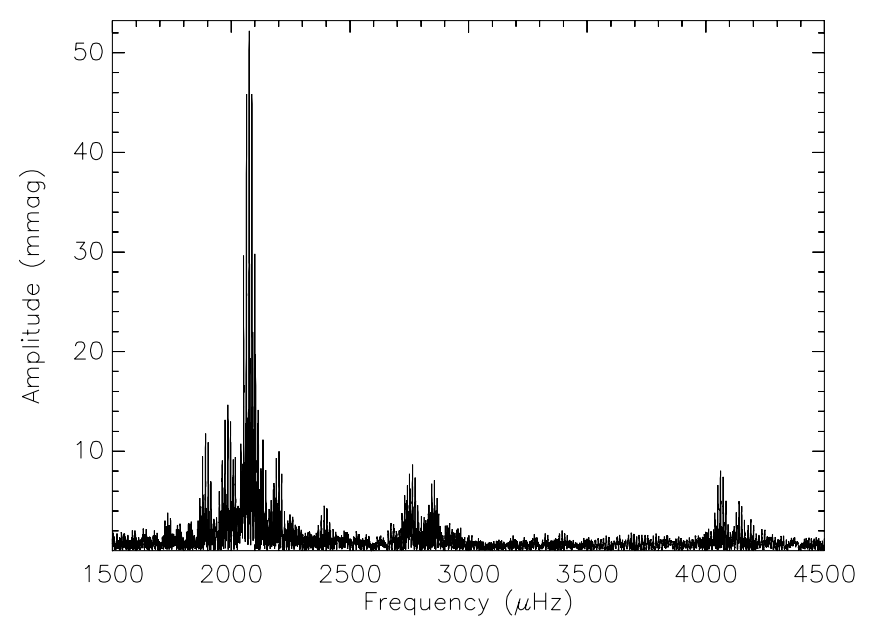

Fig. 2. Amplitude spectrum for the 7-night combined dataset in 2002 May.

Table 2. Frequencies, amplitudes and phases found for PG 1605+072 from the sine fitting procedure. The errors correspond to the formal least squares fit errors.

\begin{tabular}{lcl}
\hline \hline$v(\mu \mathrm{Hz})$ & Amplitude $(\mathrm{mmag})$ & Phase \\
\hline $1732.65 \pm 0.068$ & $3.838 \pm 0.075$ & $0.308 \pm 0.020$ \\
$1891.45 \pm 0.022$ & $11.799 \pm 0.075$ & $0.568 \pm 0.006$ \\
$1985.81 \pm 0.018$ & $14.664 \pm 0.075$ & $0.325 \pm 0.005$ \\
$2075.85 \pm 0.005$ & $52.362 \pm 0.075$ & $0.564 \pm 0.001$ \\
$2090.75 \pm 0.012$ & $21.908 \pm 0.075$ & $0.822 \pm 0.003$ \\
$2102.44 \pm 0.013$ & $19.653 \pm 0.075$ & $0.642 \pm 0.004$ \\
$2133.75 \pm 0.023$ & $11.145 \pm 0.075$ & $0.947 \pm 0.007$ \\
$2201.49 \pm 0.026$ & $9.996 \pm 0.075$ & $0.554 \pm 0.008$ \\
$2391.90 \pm 0.058$ & $4.488 \pm 0.075$ & $0.460 \pm 0.017$ \\
$2763.52 \pm 0.030$ & $8.667 \pm 0.075$ & $0.529 \pm 0.009$ \\
$2856.46 \pm 0.037$ & $7.101 \pm 0.075$ & $0.774 \pm 0.011$ \\
$4061.57 \pm 0.032$ & $8.037 \pm 0.075$ & $0.777 \pm 0.009$ \\
$4139.93 \pm 0.052$ & $4.995 \pm 0.075$ & $0.782 \pm 0.015$ \\
\hline
\end{tabular}

highest precision possible. It is possible that other frequencies could be found if we were less exigent with the accuracy of the fitting procedure. Very likely, we will improve the frequency analysis when all the data of the MSST campaign to the PG $1605+072$ is combined, once we will significantly improve on the $\mathrm{S} / \mathrm{N}$ ratio and the overall frequency resolution.

\section{Results and discussion}

The obtained frequencies, amplitudes and phases are summarized in Table 2 . The analysed frequencies are in the range of $1700-4200 \mu \mathrm{Hz}$, and the peak at $2075 \mu \mathrm{Hz}$ clearly dominates, as it can be seen in the obtained spectrum in Fig. 2 (one should keep in mind that there are several alias peaks in the spectrum, due to the daytime gaps). We have also analysed the seven time series individually, although it was decided to not include all the result tables for the sake of simplicity. While the frequency resolution of each individual time series is insufficient to resolve the closely spaced modes around the $2075 \mu \mathrm{Hz}$ mode,
Table 3. Frequency comparison with Kilkenny et al. (1999), O’Toole et al. (2002) and with the photometric $\mathrm{UV}_{B}$ study of Falter et al. (2003).

\begin{tabular}{|c|c|c|c|}
\hline$(\mu \mathrm{Hz})$ & $\begin{array}{l}v \\
(\text { Kilk.'99) } \\
(\mu \mathrm{Hz})\end{array}$ & $\begin{array}{l}v \\
\left(\mathrm{O}^{\prime} \text { Toole'02) }\right. \\
(\mu \mathrm{Hz})\end{array}$ & $\begin{array}{l}v \\
(\text { Falter'03) } \\
(\mu \mathrm{Hz})\end{array}$ \\
\hline 1732.65 & 1744.40 & - & - \\
\hline 1891.45 & 1891.42 & 1891.01 & 1891.4 \\
\hline 1985.81 & 1985.32 & 1985.75 & 1985.8 \\
\hline 2075.85 & 2075.76 & 2075.72 & 2075.9 \\
\hline 2090.75 & 2085.84 & - & - \\
\hline 2102.44 & 2103.28 & 2102.48 & 2102.0 \\
\hline 2133.75 & - & - & - \\
\hline 2201.49 & 2201.93 & - & - \\
\hline 2391.90 & 2392.04 & - & 2392.0 \\
\hline 2763.52 & 2761.30 & 2765.9 & 2763.7 \\
\hline 2856.46 & 2846.47 & - & - \\
\hline 4061.57 & 4061.81 & - & 4062.4 \\
\hline 4139.93 & 4151.48 & - & - \\
\hline
\end{tabular}

amplitude analysis over the seven nights proved to be consistent with individual runs.

\subsection{Comparison with previous studies}

In the past, PG $1605+072$ has been observed many times. Since its discovery by Koen et al. (1998), there have been several studies, photometric (Kilkenny et al. 1999; Falter et al. 2003) and spectroscopic (O'Toole et al. 2000, 2002; Woolf et al. 2002; Falter et al. 2003). So far, the reference study and the major photometric campaign has been Kilkenny et al. (1999). In the spectroscopic studies, O'Toole et al. (2002) has one of the longest time series and a very good frequency resolution, making it a good reference for frequency comparison. We have also compared our results with the latest photometric results, Falter et al. (2003).

Our frequency results are compared in Table 3. One can see that the agreement is quite good, and in fact is always below $0.7 \%$ (apart from Kilkenny et al. (1999), the relative difference is always below $0.1 \%$ ). It should be noted, however, that the frequencies compared in Table 3 might not represent the same mode - they were chosen as the nearest to our results. In fact, there are four modes - 1732, 2090, 2856, and $4139 \mu \mathrm{Hz}$ - that differ (slightly) from those found by Kilkenny et al. (1999) (and were not detected by the other two studies). This difference might arise from the different analysis methods (periodogram vs. sine fitting), or they might represent a shift in power between different and closely spaced modes, according to the beating mechanisms described in the following subsection. Despite having a relatively large amplitude (around $11 \mathrm{mmag}$ ), the $2133 \mu \mathrm{Hz}$ mode was not found in any of the other studies, implying that this is a new observed frequency, or possibly a result from some kind of artefact. 
Table 4. Amplitude comparison with three photometric studies (Koen et al. 1998; Kilkenny et al. 1999; Falter et al. 2003). The mmi units of Kilkenny et al. (1999) have been converted to mmag by the scaling factor of 1.0857. The first column serves as a mode indicator only.

\begin{tabular}{lllll}
\hline \hline Mode & Amp. & $\begin{array}{l}\text { Amp. } \\
(\text { Koen'98) } \\
(\mathrm{mmag})\end{array}$ & $\begin{array}{l}\text { Amp. } \\
(\text { Kilk.'99) } \\
(\mathrm{mmag})\end{array}$ & $\begin{array}{l}\text { Amp. } \\
\text { (Falter'03) } \\
(\mathrm{mmag})\end{array}$ \\
\hline 1732 & 3.838 & - & 1.8 & - \\
1891 & 11.799 & 6.6 & 15.1 & 4.47 \\
1985 & 14.664 & 3.8 & 3.6 & 18.79 \\
2075 & 52.362 & 63.8 & 29.7 & 28.45 \\
2090 & 21.908 & 13.4 & 3.4 & - \\
2102 & 19.653 & 20.7 & 17.3 & 16.68 \\
2133 & 11.145 & - & - & - \\
2201 & 9.996 & 1.9 & 1.2 & - \\
2391 & 4.488 & - & 2.4 & 5.8 \\
2763 & 8.667 & 5.7 & 2.0 & 13.40 \\
2856 & 7.101 & 2.2 & 2.0 & - \\
4061 & 8.037 & - & 1.3 & 4.47 \\
4139 & 4.995 & 6.8 & 1.4 & - \\
\hline
\end{tabular}

\subsection{Amplitude variation}

Pulsating sdBs in general and PG $1605+072$ in particular have shown, for some of the modes, significant amplitude variations, on several time-scales (see Kilkenny et al. 1999). In PG 1605+072, these variations were first observed in the multisite campaign of Kilkenny et al. (1999), where the amplitude of the dominant mode seems to have decreased by a factor of 2 from Koen et al. (1998), and other major variations were identified. Furthermore, O'Toole et al. (2002) discusses the amplitude variation between their two datasets, and identifies the beating between closely spaced modes as one of the explanations for the amplitude variation (on a yearly time-scale).

For instance, in the dominant mode of $2075 \mu \mathrm{Hz}$ the measured amplitude ranges from 63.8 mmag in 1997 (Koen et al. 1998) to not even being detected in 1999 (O'Toole et al. 2000). In the middle of these two results appear the 1998 results of Kilkenny et al. (1999), surprisingly with an amplitude that is roughly half the one obtained in Koen et al. (1998). Perhaps not so surprisingly, three years later (2001), the results of Falter et al. (2003) show an amplitude similar to the 1998 results. In our results, the amplitude of the dominant mode is about 52.3 mmag which, compared to the other results, is quite near from the 1997 value. These results indicate that this considerable amplitude variation seems to have a period of at least 5 years, which correspond to the interval between the two highest obtained magnitudes (63.8 mmag in 1997 and $52.3 \mathrm{mmag}$ in 2002). Whether we were lucky enough to observe twice the maximum amplitudes for this mode or are just looking at a small piece of a bigger amplitude variation, only future observations will tell.

In Table 4 we compare the obtained amplitudes with the other three photometric studies to PG 1605+072. Apart from the discussed variations in the $2075 \mu \mathrm{Hz}$ mode, one can notice
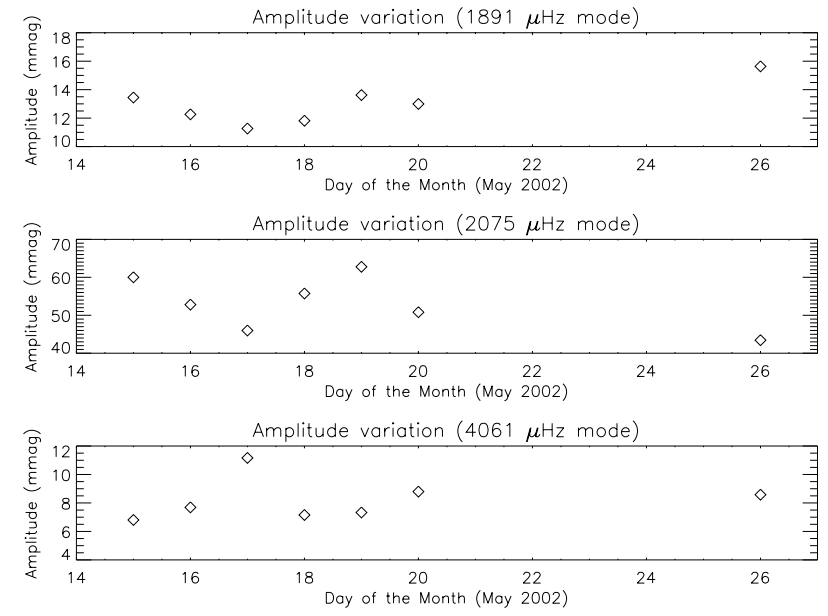

Fig. 3. Amplitude variation along the 7 nights for three of the modes. Notice the different scales of variation. Error bars are not displayed due to their reduced size, and range from $\pm 0.146 \mathrm{mmag}$ in the 18th of May and \pm 0.273 mmag in the 26th of May.

that other modes have unexpected variations (e.g. the $2763 \mu \mathrm{Hz}$ mode).

Apart from these substantial amplitude variations on a yearly time-scale, analysing the data for each night allowed us to study the amplitude variations over the seven nights, though for a limited number of modes (as a result of the poor frequency resolution for one night only). The results show a significant variation in the amplitudes, sometimes up to $25 \%$ of the mode amplitude. In Fig. 3 we plot the amplitude variation for three modes: 1891, 2075 and $2763 \mu \mathrm{Hz}$. Though some resemble a sinusoidal form, one cannot see a clear variation pattern for most of the modes. It is interesting to note that amplitude variations like the ones we report, over a time-scale in the order of days, have already been noticed in other pulsating sdB star, in the object PG 1336-018 (Kilkenny et al. 2003).

Similar to what O'Toole et al. (2002) did to test the amplitude variation in the yearly time-scale, we simulated a time series with the same length and gaps of our data. The ten strongest modes, with the parameters taken from Table 2, were the input for the artificial time series. Analysis of the individual "nights" from the simulated data revealed the same type of amplitude variations present in our data. This result leads us to conclude that, as with the yearly variations already detected, these variations in the amplitude seem to originate from a complex beating effect between the different frequencies in the spectrum.

\section{Conclusions}

Thirteen oscillation frequencies of PG $1605+072$ have been determined with an excellent accuracy from relatively short photometric time-series. Together with the complex amplitude variation this makes PG $1605+072$ one of the more interesting EC14026 pulsators to be followed closely. Except the new mode found at $2133 \mu \mathrm{Hz}$, which at present we can exclude to be produced by an artifact in the amplitude variations, the observed frequencies are in reasonable agreement with previous studies. 
Similarly to Kilkenny et al. (1999), O'Toole et al. (2002) and Falter et al. (2003), significant amplitude variations were detected, when comparing with the other photometric studies. O'Toole et al. (2002) noted that, for the detected oscillation modes there are major amplitude variations (up to $50 \%$ of the mode amplitude) that occur in a yearly time-scale - due to a beating effect between closely spaced frequencies. Not only our observations are consistent with this yearly variation of the modes amplitudes, but we have also found strong evidence that a similar effect occurs in a smaller time-scale. The analysis of individual results from our seven nights showed a clear amplitude variation and power shifting between the different modes which was confirmed by simulation.

Nevertheless, we expect that some of the open questions raised in this paper will be answered with the analysis of the full time-series analysis of MSST multi-site campaign of PG $1605+072$.

Acknowledgements. The present work was supported by a grant from Fundação para a Ciência e Tecnologia (reference $\mathrm{PESO} / \mathrm{P} / \mathrm{PRO} / 40142 / 2000$ ). We are grateful to acknowledge precious help from Rui Pereira in data acquisition and reduction. T. Pereira wishes to thank CENTRA/IST for their hosting and support. We would like to thank Anthony Lynas-Gray for useful conversations and his expertise. This paper is based on observations made at the 1-m telescope at the South African Astronomical Observatory (SAAO) in Sutherland. We wish to thank all the staff for their support and especially to Darragh O'Donaghue for his precious help and for providing his data reduction tools. We would like also to thank U. Heber, S. O'Toole and all the members of the MSST campaign team for their support.

\section{References}

Brown, T. M., Bowers, C. W., Kimble, R. A., Sweigart, A. V., \& Ferguson, H. C. 2000, ApJ, 532, 308
Brown, T. M., Ferguson, H. C., Davidsen, A. F., \& Dorman, B. 1997, ApJ, 482, 685

Charpinet, S., Fontaine, G., \& Brassard, P. 2003, in White Dwarfs, Proc. of the conference held at the Astronomical Observatory of Capodimonte, Napoli, Italy, http://www.na.astro.it/ meetings/wd2002/wd.html, ed. D. de Martino, R. Silvotti, J.-E. Solheim, \& R. Kalytis (Kluwer Academic Publishers), NATO Science Series II - Mathematics, Physics and Chemistry, 105, 69

Falter, S., Heber, U., Dreizler, S., et al. 2003, A\&A, 401, 289

Fontaine, G., Brassard, P., \& Charpinet, S. 2003, Ap\&SS, 284, 257

Heber, U., Dreizler, S., Schuh, S. L., et al. 2003, in White Dwarfs, Proc. of the conference held at the Astronomical Observatory of Capodimonte, Napoli, Italy, http://www.na.astro.it/ meetings/wd2002/wd.html, ed. D. de Martino, R. Silvotti, J.-E. Solheim, \& R. Kalytis (Kluwer Academic Publishers), NATO Science Series II - Mathematics, Physics and Chemistry, 105, 105

Kilkenny, D., Koen, C., O'Donoghue, D., \& Stobie, R. S. 1997, MNRAS, 285, 640

Kilkenny, D., Koen, C., O’Donoghue, D., et al. 1999, MNRAS, 303, 525

Kilkenny, D., Reed, M. D., O'Donoghue, D., et al. 2003, MNRAS, 345,834

Koen, C., O’Donoghue, D., Kilkenny, D., et al. 1998, MNRAS, 296, 317

O’Donoghue, D., Kanaan, A., Kleinman, S. J., Krzesinski, J., \& Pritchet, C. 2000, Baltic Astron., 9, 375

O’Toole, S. J., Bedding, T. R., Kjeldsen, H., et al. 2000, ApJ, 537, L53

O’Toole, S. J., Bedding, T. R., Kjeldsen, H., Dall, T. H., \& Stello, D. 2002, MNRAS, 334, 471

Saffer, R. A., Bergeron, P., Koester, D., \& Liebert, J. 1994, ApJ, 432, 351

Schechter, P. L., Mateo, M., \& Saha, A. 1993, PASP, 105, 1342

Sperl, M. 1998, http://dsn.astro.univie.ac.at/ period98

Woolf, V. M., Jeffery, C. S., \& Pollacco, D. L. 2002, MNRAS, 329, 497 